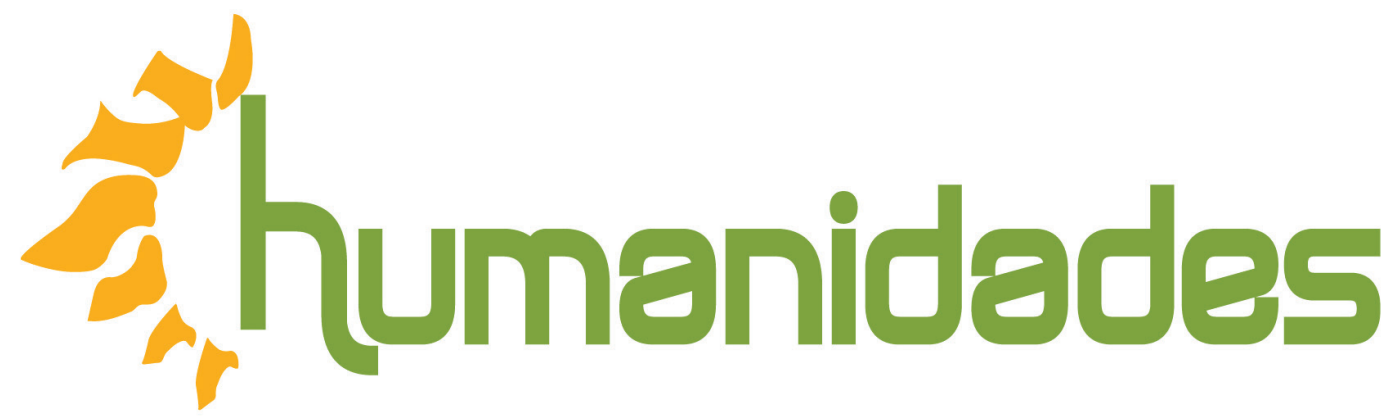

Revista de la Escuela de Estudios Generales, Universidad de Costa Rica

Enero-julio, $2019 \cdot$ Volumen 9, número $1 ・$ EISSN 2215-3934 • pp.1-23

Recibido: 1-Agosto-2018 Aceptado: 23-Agosto-2018

\title{
Arte público en la Sede del Atlántico, Universidad de Costa Rica (2014-2017)
}

DOI: https://doi.org/10.15517/h.v9i1.35285

\section{Pablo Bonilla Elizondo}

Doctor en artes, profesor en el Escuela de Artes Plásticas de la Universidad de Costa Rica, Costa Rica.

Correo electrónico: pablobonillaucr@gmail.com

Todos los derechos reservados. Universidad de Costa Rica. Esta revista se encuentra licenciada con Creative Commons. Reconocimiento-NoComercial-SinObraDerivada 3.0 Costa Rica. Correo electrónico: humanidades@ucr.ac.cr/ Sitio web: http: //revistas.ucr.ac.cr/index.php/ humanidades 


\title{
Arte público en la Sede del Atlántico, Universidad de Costa Rica (2014-2017)
}

\section{Resumen}

Este artículo presenta un conjunto de proyectos de arte público realizadas en la Sede Universitaria del Atlántico, en Turrialba, desde la implementación de la carrera de Diseño Gráfico en dicho recinto. Los proyectos efectuados han transformado el espacio llenándolo de color y sentido, además, han puesto en escena los principales íconos de la cultura nacional y turrialbeña. Los proyectos también han permitido problematizar el presente de la comunidad. De esa forma, se ha logrado hacer un homenaje y rescate de la cultura e identidad local a través de una mirada crítica del presente.

Además, en este artículo se exploran los conceptos de Arte público: nuevo género y el Derecho a la Ciudad como marco para fundamentar las intervenciones. Ambas nociones permiten reflexionar sobre la presencia de arte público en los campus, recintos o sedes universitarias en el marco de la formación humanista.

\section{Public Art at Sede del Atlántico, University of Costa Rica (2014-2017)}

\begin{abstract}
This paper presents a set of public art projects carried out in the University of Costa Rica's Sede del Atlántico, in the city of Turrialba, since the implementation of the Graphic Design major in that place. The projects carried out have transformed the space filling it with color and meaning. They have also staged the main icons of both national culture and the culture of Turrialba's inhabitants. Furthermore, the projects have allowed to problematize the present of the community. In this way, a tribute to and a rescue of the local culture and identity have been achieved through a critical view of the present.

In addition, this paper considers the concepts of New Genre Public Art and the Right to the City as background for the interventions, for this ideas allow to reflect on the presence of public art in the university venues in the framework of humanist education.
\end{abstract}

Keywords:

public art, university life style, public space, humanism, participation 


\title{
1. Arte público y espacio político
}

A finales de la década de los ochenta, teóricas del arte y artistas norteamericanas vinculadas al feminismo y activismo político, como Suzanne Lacy, Mary Jane Jacobs y otras (Lacy, 1994) empezaron a utilizar con frecuencia el concepto de New Genre Public Art [Arte Público: Nuevo Género] con el propósito fundamental de situar una serie de nuevas prácticas distantes de la idea tradicional del arte público, entendido como grandes "obras de Arte” emplazadas en el espacio público. Así define Lacy (1994) este Nuevo género de arte público:

\begin{abstract}
Unlike much of what has heretofore been called public art, new genre public art-visual art that uses both traditional and nontraditional media to communicate and interact with a broad and diversified audience about issues directly relevant to their lives - is based on engagement. (...) Attacking boundaries, new genre public artist draw on ideas from vanguard forms, but they add a developed sensibility about audience, social strategy, and effectiveness that is unique to visual art as we know it today.
\end{abstract}

[A diferencia de lo mucho que hasta ahora se ha llamado arte público, el nuevo género de arte público — arte visual que utiliza medios tradicionales y no tradicionales para comunicarse e interactuar con un público amplio y diverso sobre temas directamente relevantes para sus vidas - se basa en el compromiso. (...) $\mathrm{Al}$ atacar los límites, los artistas públicos de este nuevo género recurren a ideas vanguardistas, pero agregan una sensibilidad desarrollada sobre la audiencia, la estrategia social y una efectividad que es única en el arte visual tal como lo conocemos hoy $]^{1}$ (p. 19-20).

Este nuevo género, como es patente, se opone a la idea tradicional de arte público, que incluía de forma limitada murales, propaganda, monumentos conmemorativos y esculturas de los grandes artistas modernos que redimensionaban y adaptaban sus procesos creativos al espacio público y que, naturalmente, se encontraba anclada en un marco institucional, normativo e histórico que evidenciaba una estructura de poder claramente jerarquizada, donde el público figuraba como un simple consumidor pasivo. En ese sentido, esta noción tradicional de arte público, 
entendido como el emplazamiento de obras artísticas en el entorno urbano desde las posiciones de poder — en la lógica perpetuación de ese régimen—, era entendido por este conjunto de autoras como una forma de codificación simbólica que restringía a las ciudades bajo una funcionalidad comercial, didáctica o de cohesión ideológica.

Por ejemplo, Rosalind Krauss en su ensayo El campo expandido de la Escultura (1996) aborda con exactitud la lógica propia de los monumentos conmemorativos que coronan la figura del prócer de la ciudad y la posterior aparición de la escultura de arte moderno en contraste con la arquitectura burocrática estadounidense.

El Arte público: nuevo género desafía la progresión de la historia oficial del arte público atada a las normativas institucionales y las jerarquías gubernamentales (lo que no ha significado su absoluta independencia a ella; al contrario, se podría decir que ha impulsado la transformación de esas normativas para el cuestionamiento de las formas en que se promueve el arte y la cultura comunal desde la gobernanza). En un principio, se fundamenta en una serie de antecedentes políticos, artísticos y culturales ligados al postestructuralismo, el marxismo frankfurista, el feminismo, el activismo político y las reivindicaciones sociales, raciales y étnicas que configuran sus prácticas en torno al cuestionamiento de las relaciones de poder, el determinismo de las estructuras sociales y las posibilidades de acción de la ciudadanía en la vida política. En ese sentido, el Arte público: nuevo género asumía el compromiso de cuestionar "lo público" y promover un rol activo de la ciudadanía en la producción cultural y simbólica de su ciudad o comunidad, fuera de los relatos heroicos e interioristas de "los grandes maestros del arte" o los escultores monumentales.

En este marco, fácilmente podemos ubicar un conjunto de prácticas artísticas que reconocen la importancia de someterse a procesos de investigación más rigurosos en cuanto al análisis del contexto, con equipos de trabajos interdisciplinares que den cuenta de las complejidades epistemológicas de lo público y lo político. Además, estas prácticas se comprometen con la inclusión de los espectadores o beneficiarios en los proyectos, no solo en su diseño, sino también en todo su proceso de planificación y realización. Este último punto permite reconfigurar a través del arte las relaciones subjetivas y cívicas con el espacio político y convertir esa relación en el propósito primero de la manifestación artística, más allá de sus resultados materiales (sea este un mural, una escultura o una instalación multimedia). 
Como señala Suzanne Lacy (1994):

The inclusion of the public connects theories of art to the broader population: what exist in the space between the words public and art is an unknown relationship between artist and audience, a relationship that may itself become the artwork.

[La inclusión del público conecta teorías del arte con una población más amplia; lo que existe en el espacio entre las palabras público y arte es una relación desconocida entre el artista y el público, una relación que puede convertirse en la obra de arte $]^{2}$ (p. 20).

Apoyados en estas concepciones, el mural Parcelas de Aire, desarrollado en la Sede del Atlántico en el año 2015, ha buscado eliminar esa distancia "metafísica" entre artista, espacio socio-político y espectadores o beneficiaros por medio de un proceso de investigación interdisciplinar y colaborativo (que detallaremos en la metodología) capaz de establecer un conjunto de relaciones significativas que dotan al mural de una identidad local e histórica a partir de un involucramiento real y no de una narrativa didáctica.

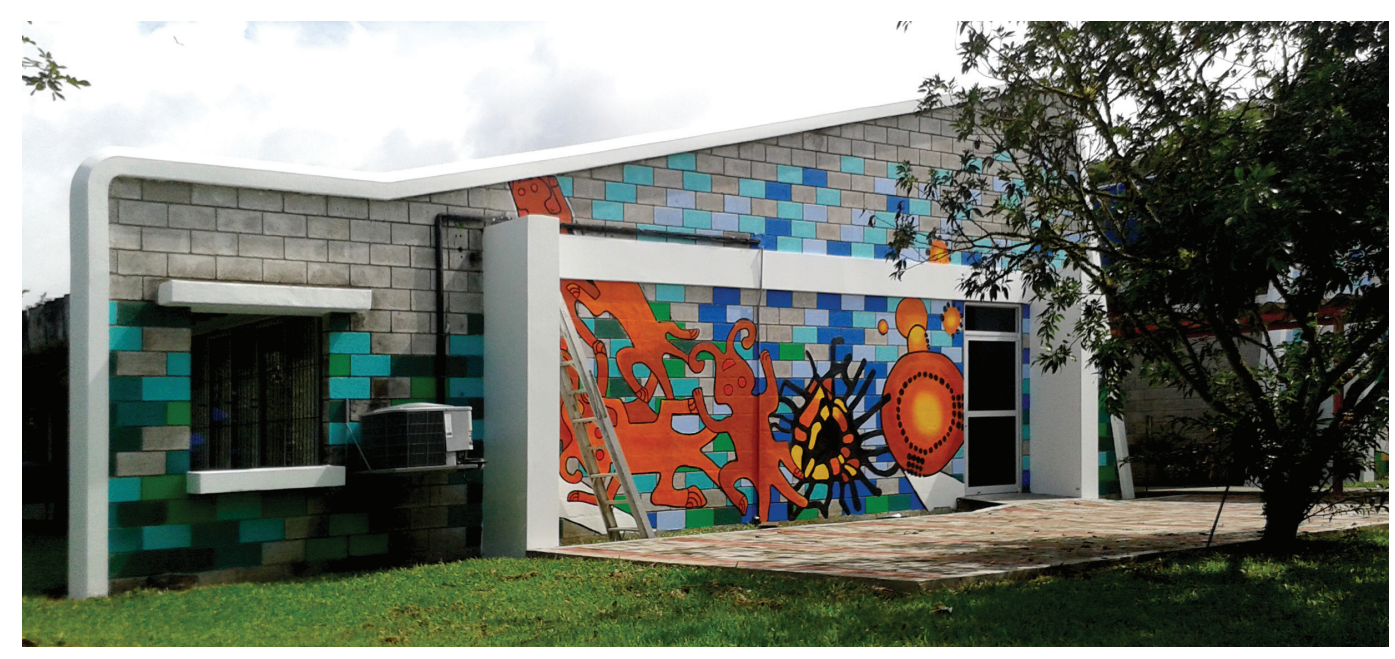

Foto \#1:

Mural Parcelas de Aire, lado A.

CPablo Bonilla Elizondo 


\section{Universidad humanista y espacio universitario}

En el campus universitario, las metodologías participativas y los procesos de investigación propios del Arte público: nuevo género se consideran coherentes con las ideas que fundamenta la universidad humanística, la cual entiende la formación universitaria como una formación integral, crítica y consciente de la realidad social $\mathrm{y}$, por tanto, debe comprender su espacio físico como un entorno capaz de construir relaciones potentes, diversas y complejas entre distintas subjetividades, formas de hacer, sentir y pensar.

Dentro de ese espíritu humanista, el campus universitario no puede ser entendido como un conglomerado de aulas más o menos ordenado donde se ejerce la docencia de forma jerarquizada. Debe también ofrecer a los estudiantes, administrativos y docentes diferentes estímulos a diferentes niveles. Las cafeterías, zonas verdes, plazas, pretiles, etc., no solo son espacios para la recreación, el deporte y el descanso (sumamente importante), sino también son espacios de reflexión, estudio, lectura y discusión que permiten confrontar, con amigos y compañeros de diversas carreras, edades y formaciones, lo aprendido en clases. El arte público, así como una gestión cultural activa que lleve arte en sus distintas manifestaciones (música, danza, etc.) a todos estos sitios, potencia el entorno universitario como un conjunto de espacios para sentir, re-crear y pensar que hacen justicia a los propósitos que una universidad humanista proyecta para la transformación social.

Sobre las posibilidades y potencialidades de estas transformaciones, donde el campus universitario se presenta como una urbe modelo o, mejor dicho, un modelo de urbe, es importante mencionar los postulados sobre el Derecho a la Ciudad propuestos por Henri Lefebvre en 1968 (1978). Estos postulados reconocen la ciudad y el espacio público no como un contenedor de la vida de las personas regulado desde el poder gubernamental, sino como un espacio político generador de múltiples conflictos, de encuentros y desencuentros donde el poder se ejerce en constante tensión y de manera asimétrica. 
Ante ello, este espacio debe considerarse como un escenario de contante reelaboración para el arte a partir del cual se pueda dar paso a formas más democráticas de acción política por medio de un ejercicio activo de una ciudadanía que constantemente construye, reconstruye y resemantiza la ciudad. Ante ello, este espacio debe considerarse como un escenario de contante reelaboración para el arte a partir del cual se pueda dar paso a formas más democráticas de acción política por medio de un ejercicio activo de una ciudadanía que constantemente construye, reconstruye y resemantiza la ciudad.

A partir de estas ideas, es elemental concebir el campus universitario como un espacio dinámico y en constante configuración que posibilite a estudiantes y docentes apropiarse y ser parte de él. A la larga, desarrollar un sentido de pertenencia no sólo posibilita un mejor cuido de la infraestructura y a los espacios públicos, sino que permite la construcción de una conciencia ciudadana sobre el ser y el hacer en comunidad en el entorno de lo político que, como es sabido, no se reduce a un proceso electoral u otro, sino que densifica la vida en la polis en todo su espesor y complejidad.

De esta forma, el Arte público: nuevo género se presenta como uno de los elementos complejos de la ciudad que cuestiona e incita a reflexionar sobre las relaciones que se establecen entre la comunidad y su espacio. No es propio pensar que esto debe ser ajeno a cualquier recinto universitario; por más pequeño que sea, el desarrollo de actividades culturales y artísticas potenciará simbólicamente sus dimensiones.

En la Sede Rodrigo Facio, la presencia del arte público no ha sido extraña. En ella se vislumbran monumentos conmemorativos, principalmente en el formato de bustos, obras murales de Eduardo Torijano (quien da impulso al muralismo dentro de la infraestructura universitaria), esculturas abstractas y figurativas (donde conviven múltiples proyectos de graduación de la Escuela de Artes Plásticas y obras de artistas con gran trayectoria como José Sancho y Leda Astorga). También es importante señalar que el arte público ha acompañado el crecimiento de la Ciudad de la Investigación y del Jardín Botánico Lankester, donde las esculturas dialogan de una forma muy especial con la naturaleza. 
Respecto a las sedes regionales, resulta oportuno mencionar a la Sede de Occidente, cuyo impulso al arte público se ha dado desde la apertura de artes plásticas y artes gráficas, sobre todo a partir del proyecto de Acción Social "Poemas Gráficos", lo que evidencia el impacto que la enseñanza artística es capaz de generar dentro del campus.

Sin embargo, los procesos participativos para el arte emplazado en el campus siguen siendo escasos o inexistentes desde la gestión institucional, principalmente para obras de gran envergadura, como si la participación de la comunidad universitaria no fuera requerida para proyectos de gran magnitud presupuestaria. Sin embargo, estas metodologías han sido asumidas con mucho entusiasmo por grupos artísticos estudiantiles, como el Colectivo Artemutación UCR y la EFE, quienes han asumido la responsabilidad de la activación socio cultural del campus universitario Rodrigo Facio desde prácticas efímeras, lúdicas o performáticas. Gracias a ellos, a la presión que ejerce los estudiantados desde sus propios intereses, ya se empieza a vislumbrar en docentes y administrativos un cambio de actitud con respecto a cómo abordar el problema del espacio público universitario a partir de ideales humanistas transdisciplinares, que posibiliten la convivencia de todas estas modalidades como un rico paisaje de diversidad.

Elpropósito fundamental del proyecto mural Parcelas de Aire era, consecuentemente, articular los procesos participativos con una obra mural en la Sede del Atlántico. Se pretendió, desde un primer momento, no oponer de forma simplista el concepto de arte público tradicional con el de nuevo género, sino, al contrario, se buscó afirmar la posibilidad de su articulación. No es la intención de este artículo negar la importancia de las obras monumentales o abstractas en el espacio público, sino evidenciar que estas pueden ser concebidas desde metodologías de investigaciónparticipación y que, por lo tanto, tales prácticas no son excluyentes. 


\section{Algunos elementos del contexto y la integración del mural Parcelas de Aire}

La Sede del Atlántico fue fundada el 22 de agosto de 1973 como parte de la política de regionalización y descentralización de la Universidad de Costa Rica. Los terrenos donde se encuentra la universidad fueron comprados al Centro Agronómico Tropical de Investigación y Enseñanza (CATIE) en 1973. Esta finca, conocida como "La Hulera", constituyó la sede de la empresa inglesa Rubber Reserve Company en los años 40 y luego fue vendida al CATIE.

Bajo el punto de vista arquitectónico y patrimonial, los edificios que se construyeron en esta finca obedecían al concepto de una estación experimental agrícola (especialmente de hule) en un ambiente tropical húmedo. Estos inmuebles forman un conjunto arquitectónico propio de la década de los cuarenta, integrado por lo que hoy es el Museo Omar Salazar, la etapa básica de música y las oficinas de los profesores. Están construidos con madera, con techos de dos aguas y cuentan con muy buena ventilación para lidiar con el calor y la humedad de la zona. Estos edificios fueron declarados patrimonio arquitectónico en 1995. El conjunto forma la fachada de la Sede, la cual le imprime una identidad particular, muy diferente de otras sedes regionales de la universidad.

Las edificaciones de la segunda etapa, de construcción posterior, consisten en un pabellón para la administración, otro para la docencia, cubículos de profesores, un pabellón de aulas, una biblioteca y una cafetería. Los inmuebles datan de los años ochenta y es la primera gran inversión que hace la Universidad de Costa Rica en infraestructura en Turrialba. El diseño arquitectónico que se estableció es muy moderno y apropiado. No tiene relación alguna con los edificios históricos de la antigua hulera.

El tercer grupo lo conforma una construcción reciente, la cual se ha destinado, entre otras cosas, a los programas de educación indígena. Por ello es conocido como "módulo de educación indígena". Se trata de un edificio moderno y ventilado que data del 2013. Por sus características, forma un grupo aparte. 
El mural Parcelas de Aire, construido en diciembre del 2014 e inaugurado el 26 de marzo del 2015, es un puente que une la etapa dos, es decir, los edificios de 1980 y el módulo de educación indígena del 2013. Bajo la óptica de la identidad, el mural pretende establecer un diálogo entre el pasado y el presente.

Si bien es cierto que el sitio arqueológico de Guayabo es un parque nacional y monumento arqueológico desde el 23 de agosto de 1973 bajo la ley \#161, también es cierto que para muchos costarricenses está muy lejos de su imaginario y, más aun, de su vida cotidiana. Nada más ilustrativo que frente al mural muchos estudiantes se preguntan de dónde son esos "bichos". La presencia estilizada de los petrógrafos de Guayabo y algunas de sus imágenes más reconocidas: el jaguar, los montículos, la mal llamada "araña", que para algunos es obvia y para otros no tanto, ubican al mural en un lugar histórico y geográfico preciso, el cantón de Turrialba, y lo conectan con su presente.

En cuanto a los petroglifos, el arqueólogo Carlos Aguilar señala: "El elemento constitutivo más importante en el traslado de los petroglifos es la espiral, un estudio cuidadoso podría arrojar alguna luz sobre su significado" (1972, p. 126). Para la concepción de este mural y la configuración de sus elementos de diseño, se partió de la apropiación de las espirales recurrentes en los petroglifos, además de vistas áreas de Guayabo que supusieron una estructura intermedia entre el fondo y las figuras. Así se establecieron tres niveles articulados: las formas de animales estilizadas como primer plano, los petroglifos espirales y las vistas áreas en segundo plano y, por último, el paisaje turrialbeño.

La geografía está presente por medio de una representación del volcán Turrialba, tantas veces fotografiado y dibujado hasta el "cliché", pero que en este mural toma otra dimensión más estética por medio de los siguientes elementos: (1) el uso del color, el cual tiene gran relevancia al alegorizar el clima tropical del atlántico por medio de una gama de colores cálidos (rojos, naranjas, amarillos y verdes cálidos); y (2) el aprovechamiento de las características constructivas de la fachada como patrón para una aplicación del color de forma consecuente con los bloques de cemento expuestos propios del inmueble. Este último detalle logró una mayor integración entre el mural y el edificio, ya que buscó evitar cierta sensación de superficialidad. En este caso, el mural pareciera nacer del inmueble. 
El mural también incluye un fragmento del poema "Nocturno sin patria" (2012) del poeta turrialbeño Jorge Debravo. Estos versos no solo son un homenaje más al poeta y a su reconocimiento como parte integral del legado cultural turrialbeño que se ha extendido a toda Costa Rica, sino que trasmiten una profunda sed de justicia social, sobre todo en el campo. Estos versos son propios de los movimientos que en la época de Debravo demandaban la distribución de las tierras a manos campesinas, pero hoy, claramente, siguen siendo relevantes en el marco de un capitalismo avanzado donde la producción agrícola se centra en la exportación y explotación territorial, la cual se opone a la conciencia campesina que requería una relación sostenible entre producción y consumo para el beneficio de todas las partes. Así, al contrario que dibujar el rostro del poeta -recurso ya en exceso utilizado-, se eligió la palabra, en tanto transmite efectivamente una problemática de la zona que ya era preocupación del poeta y que hoy se presenta vigente, sobre todo para la zona en la cual se emplaza la Sede del Atlántico, inspiración clara del autor. A continuación, se adjunta el poema completo:

"Nocturno sin patria"

Jorge Debravo

Yo no quiero un cuchillo en manos de la patria

Ni un cuchillo ni un rifle para nadie:

La tierra es para todos,

Como el aire.

Me gustaría tener manos enormes, Violentas y salvajes, Para arrancar fronteras una a una

Y dejar de fronteras solo el aire

Que nadie tenga tierra

Como se tiene traje:

Que todos tengan tierra

como se tiene el aire

Cogería las guerras de las puntas

Y no dejaría una en el paisaje

Y abriría la tierra para todos

Como si fuera el aire... 
Que el aire nos es de nadie, nadie, nadie...

Y todos tienen su parcela de aire.

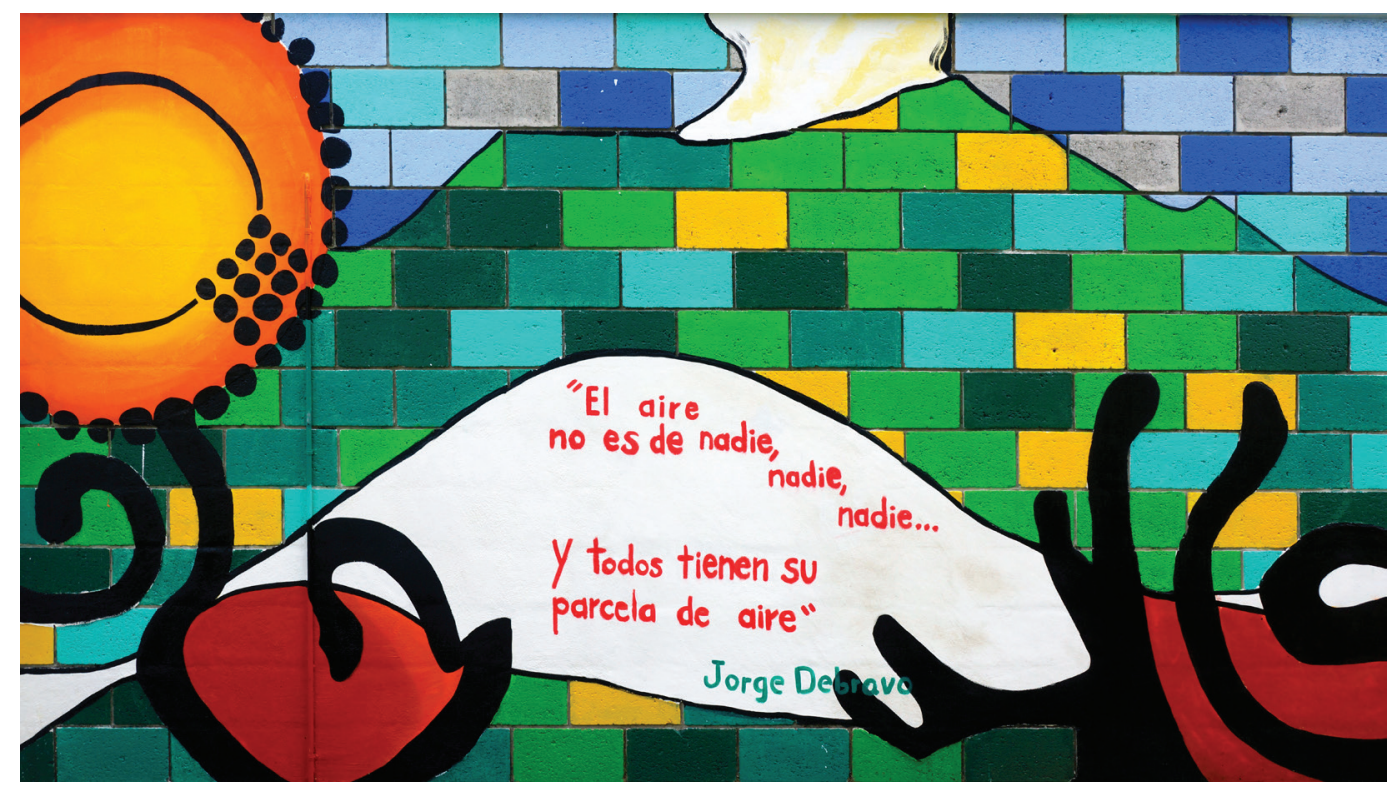

Foto \#2.

Mural Parcelas de Aire, detalle sobre poema de Jorge Debravo.

CPablo Bonilla Elizondo

Para el proceso del diseño y realización, se optó una metodología de investigaciónparticipación-acción propia de pensadores como Paulo Freire (1998) y Orlando Fals Borda (2003). Se inició por reconocer las condiciones intrínsecas (condiciones materiales, diseño arquitectónico e incidencia de agua y lluvia) y extrínsecas del inmueble y la pared (tránsito peatonal, perspectivas y angularidad de la mirada, paisaje aledaño, función social del inmueble y zonas cercanas). También se realizó una visita guiada al Sitio Arqueológico de Guayabo, donde se tomaron fotografías de los petroglifos y se complementaron con el estudio de diversas publicaciones sobre el tema. Entre ellas, cabe resaltar la publicación $w$ de las autoras Ana Cecilia Arias, Floria Castrillo y Grace Herrera, editada por la Vicerrectoría de Acción Social de la Universidad de Costa Rica (2012). Con el apoyo de los compañeros docentes de la Sede del Atlántico, el proceso de diseño se concluyó con la selección de un verso de Jorge Debravo que tituló el mural. Todo el primer proceso de investigación y diseño fue remitido al Consejo de Sede para su retroalimentación y discusión, lo cual llevó a la inclusión de sugerencias y recomendaciones previo a su aprobación. 
Después de la aprobación del diseño por parte del Consejo de Sede, se inició el proceso para su realización. Así, se conjuntó un equipo conformado por un albañil, estudiantes de TCU de la Sede del Atlántico y la Sede Rodrigo Facio (Marcela López Esquivel, Diana Patricia Murillo Chavez, María Fernanda Savrez Vega, Carol Cristina Solano Nájera y Leyla Vargas Zaxár) y el artista visual Orlando Guier Oreamuno. La Sede del Atlántico realizó un trabajo de gestión preciso para permitir que las obras se realizaran fluidas, sin pormenores, con todos los aspectos de seguridad y según las etapas previstas dentro del cronograma presentado. Esto, sin lugar a dudas, merece ser mencionado, dado que en estos proyectos complejos la articulación entre el proceso de investigación y de gestión administrativa es fundamental para llevar a buen puerto estas iniciativas.

El proyecto fue entregado para la segunda semana de diciembre del 2014 con un informe técnico que plantea estrategias de mantenimiento y posibilita una restauración sin complicaciones en caso de accidentes o imprevistos. Este informe no solo es un documento necesario, sino que forma parte de un conjunto de buenas prácticas en la profesión artística que permiten desestabilizar el mito que supone la permanencia inmutable e inmaculada de las obras de arte público.

En síntesis, estas fueron las etapas de realización del mural:

1. Investigación de la cultura y el entorno turrialbeño, visitas guiadas al Sitio Arqueológico de Guayabo, recopilación y procesamiento de fuentes y referencias bibliográficas y discusiones y mesas de trabajo con docentes y estudiantes de la Sede.

2. Diseño de una propuesta inicial para su aprobación en las instancias pertinentes. Se añadieron modificaciones y sugerencias dadas por los profesores de la Sede del Atlántico y se aprobó el diseño final.

3. Gestión administrativa preliminar para la realización del mural, compra de materiales, contratación del personal, reserva de dormitorios, bodegaje, etc.

4. Traslado del diseño al muro y delineado en tiza y en pintura. 


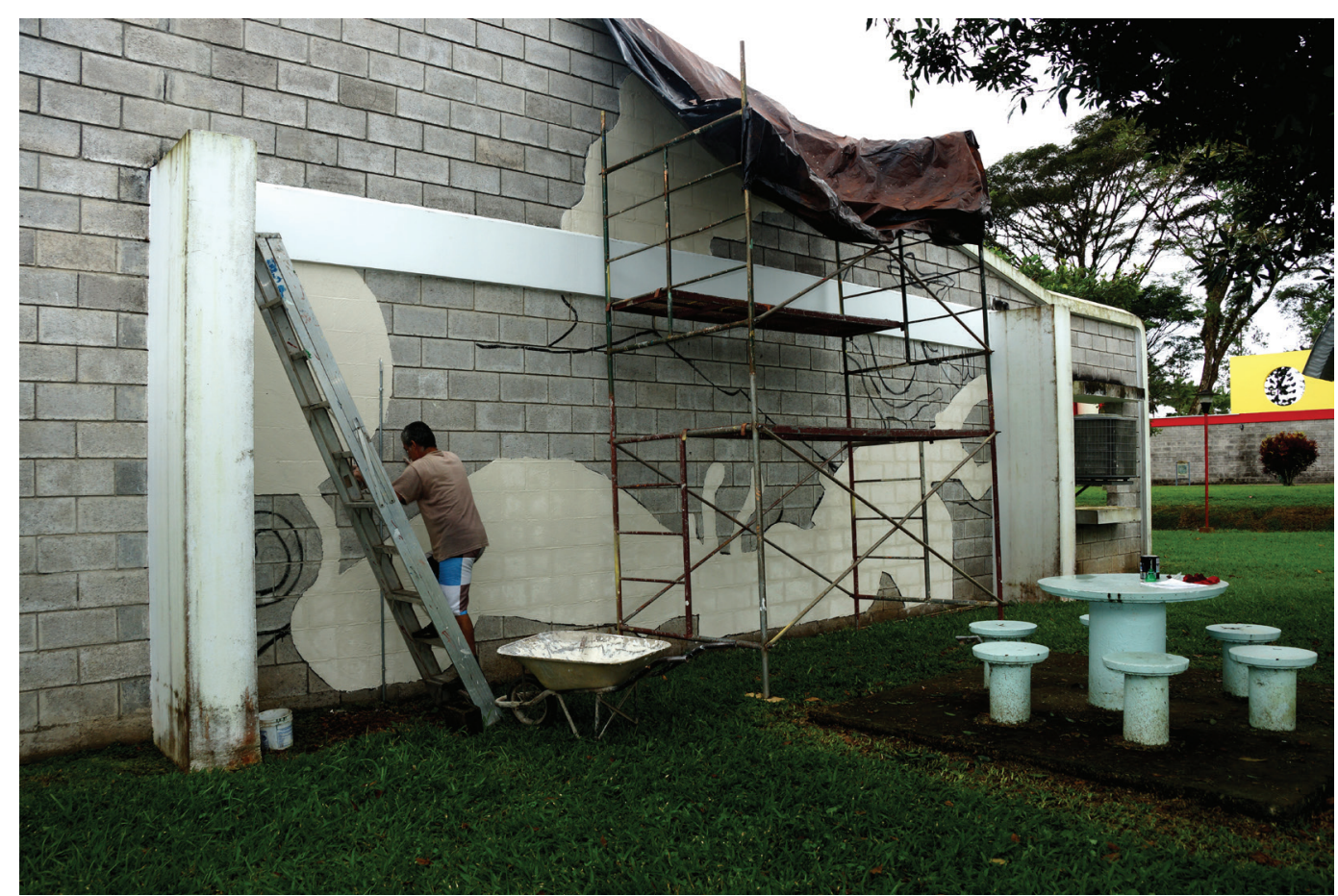

Foto \#3.

Mural Parcelas de Aire, aplicación de repello. CPablo Bonilla Elizondo

5. Realización del repello selectivo para separar fondos de figuras.

6. Pintura de las zonas de color del fondo realizadas por los estudiantes del TCU.

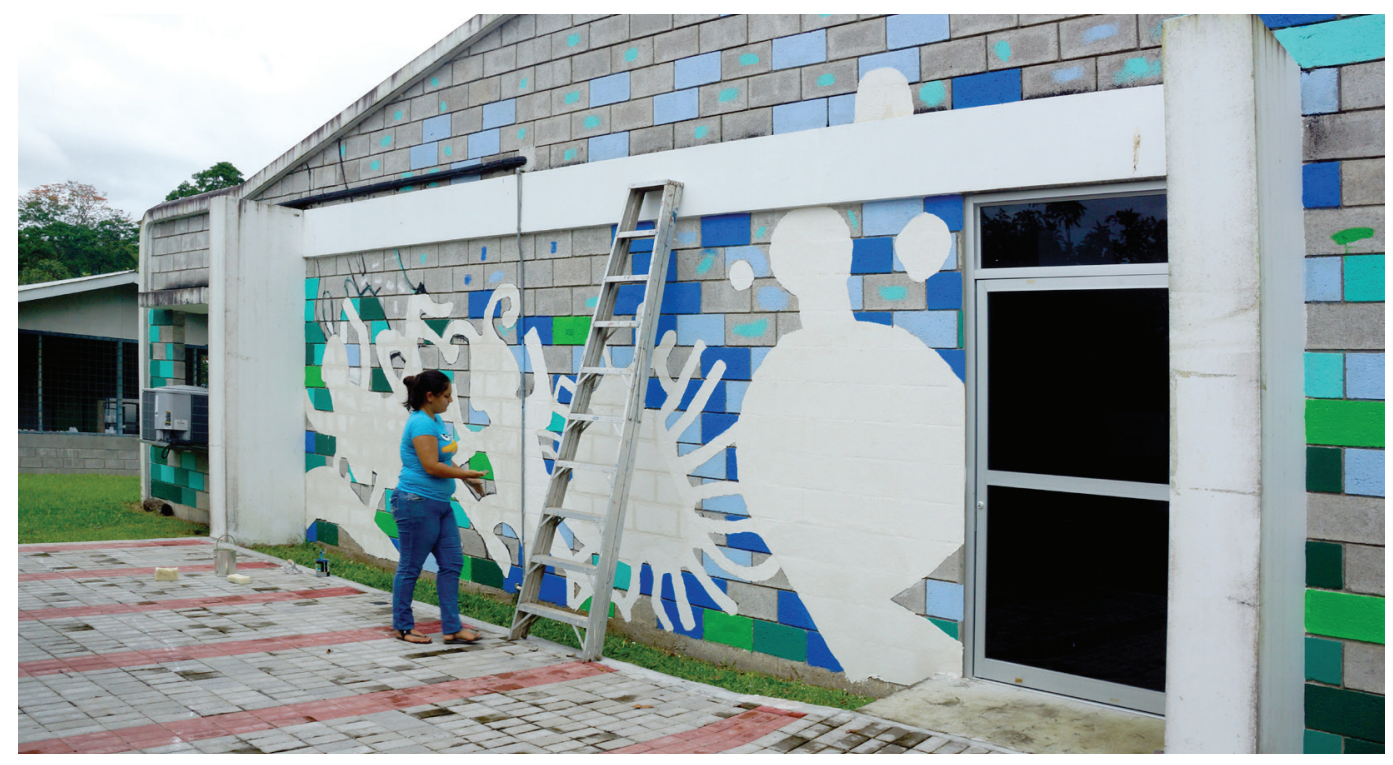

Foto \#4.

Mural Parcelas de Aire, participación estudiantil. CPablo Bonilla Elizondo 
7. Detallado y delineado de las figuras (planos primeros y segundos).

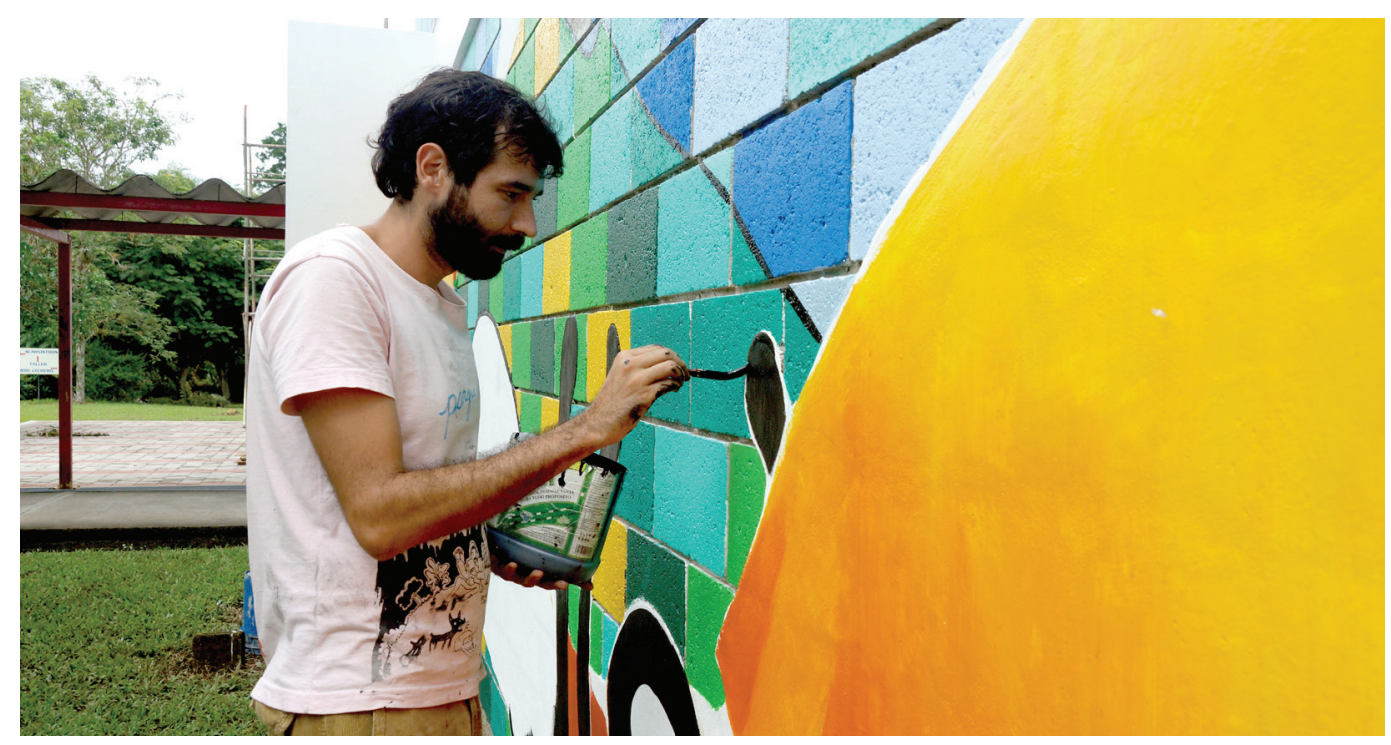

Foto \#5.

Mural Parcelas de Aire, deliniado final. CPablo Bonilla Elizondo

8. Finalización y aplicación de una capa de sellador contra agua.

9. Entrega del mural y su informe técnico (segunda semana de diciembre del 2014).

10. Se conoce en Consejo de Sede el informe técnico y se recibe de forma satisfactoria el proyecto el día miércoles 3 de marzo del 2016.

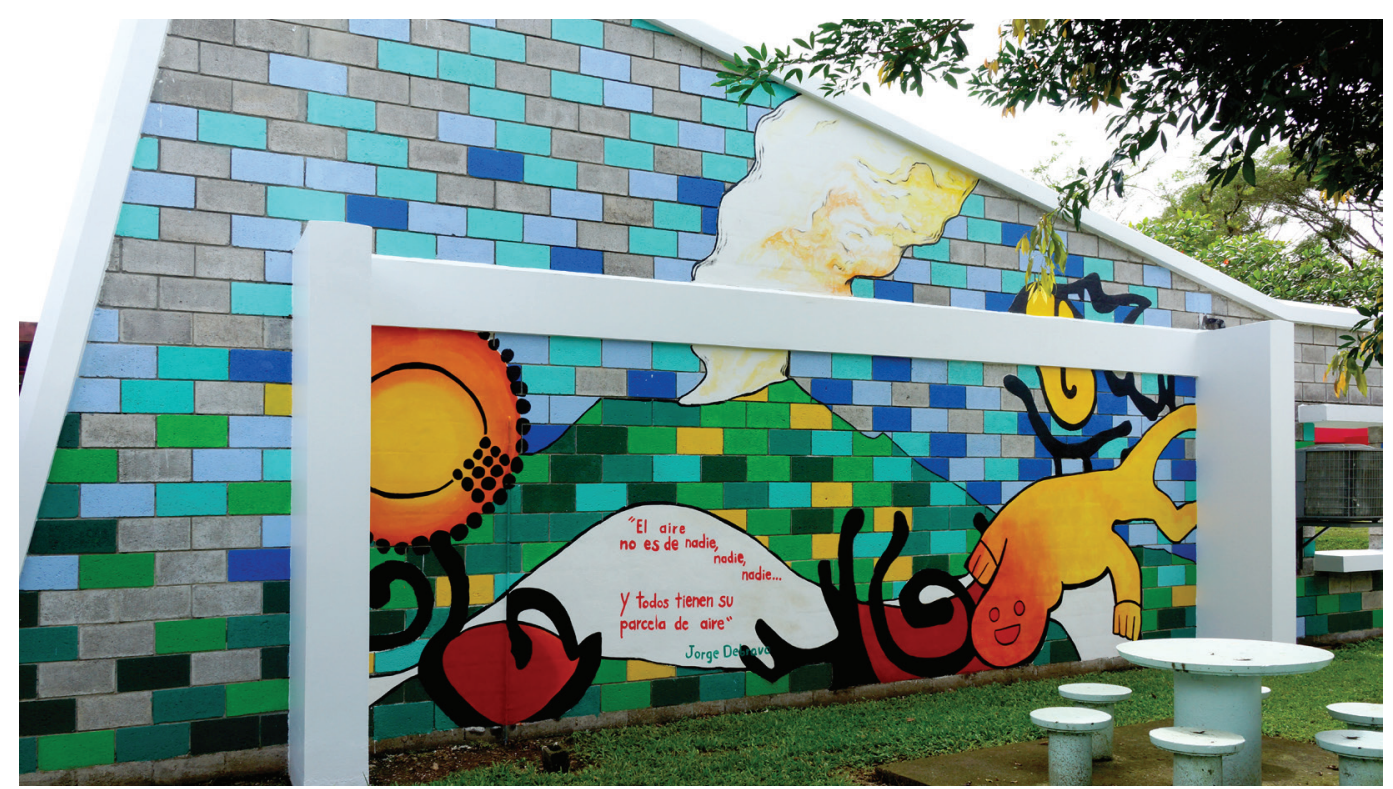

Foto \#6:

Mural Parcelas de Aire, lado B.

CPablo Bonilla Elizondo 
Se puede concluir, por tanto, que el proyecto mural Parcelas de Aire se presenta como una experiencia socio-cultural compleja que bajo el modelo de investigaciónparticipación responde a cabalidad con el entorno y la función humanista que recae en el quehacer de la Universidad de Costa Rica. Este proyecto mural, por consiguiente, afirma por su propia identidad que el arte público en el ámbito universitario puede cumplir con propósitos más allá de mejorar el entorno, sobre todo cuando asume la transdisciplinariedad y la gestión cooperativa como motor de sus acciones.

En específico, los propósitos cumplidos por este proyecto se pueden resumir así: afirma la condición social del campus universitario; acerca a los estudiantes, profesores y administrativos a su herencia prehispánica, la historia, la cultura y el paisaje de Turrialba; entraña lazos identitarios; mejora el entorno; y promueve el uso de los espacios públicos aledaños.

\section{II Encuentro de Arte Público: Diversidad Metropolitana}

Sin lugar a dudas, el mural Parcelas de Aire representó para el recinto de Turrialba de la Universidad de Costa Rica la incursión en metodologías participativas transdisciplinares volcadas a afectar positivamente dicha comunidad de aprendizaje que, a su vez, fueron coincidentes con la apertura de la carrera de Diseño Gráfico en tal sede en el 2015, lo cual permitió profundizar en estas dinámicas. Por ello también, ya consolidada la carrera para el 2017, al organizar desde la Escuela de Artes Plásticas de la Sede Rodrigo Facio el II Encuentro de Arte Público: Diversidad Metropolitana, no se dudó en emplazar en Turrialba una serie de talleres, entre ellos el impartido por el Doctor Christof Göbel y el Master Olar Zapata, ambos académicos respetados de la Universidad Autónoma Metropolitana de México y vinculados al Posgrado en Estudios Urbanos que imparte dicha institución. 
En términos generales, el II Encuentro de Arte Público: Diversidad Metropolitana se realizó de forma conjunta entre el Departamento de Evaluación del Diseño en el Tiempo de la Universidad Autónoma Metropolitana, la Escuela de Artes Pláticas de la Sede Rodrigo Facio, las coordinaciones de la carrera de Diseño Gráfico y Plástico en la Sede del Atlántico, la Sede de Occidente y la Intersede de Alajuela de la Universidad de Costa Rica, entre el 2 y el 13 de octubre de 2017. El Encuentro, más que intervenir en lo interno del campus - como se había hecho previamente-, se centró en problematizar las relaciones complejas entre las diferentes metrópolis y las sedes universitarias, pues contempló la articulación entre sus espacios inmediatos y sus conexiones más distantes en función de otras formas de desplazamientos e imaginarios.

Más allá de cualquier forma de diagnóstico o respuesta objetiva a los conflictos urbanos, este II Encuentro de Arte Público se enfocó en los imaginarios, en las conexiones virtuales $y$, sobre todo, en la potencia discursiva del arte para subvertirlos en formas de resistencia, dada una condición problemática de la ciudad costarricense que no sólo afecta el plano material de la estructura social, sino también la vida misma. En ese sentido, el proyecto se centró en lo biopolítico mediante las interrogantes: ¿cómo es posible estetizar la vida en este entorno hostil?, ¿cómo podemos vivir juntos? y ¿cómo podemos conducirnos de la forma más virtuosa posible en la experiencia de la ciudad entre los trazos de esos conflictos que la marcan?

En específico, el taller impartido en conjunto por Christof Göbel y Olar Zapata se denominó Análisis, experimentación e intervención del espacio público y, como su nombre lo indica, partía de actividades de diagnóstico (visualización de referencias, mapeos y discusiones) para, posteriormente, diseñar y llevar a cabo un conjunto de intervenciones en el centro de Turrialba. Dichas intervenciones se centraron en la construcción crítica de experiencias vitales y relacionales, articuladas con el pasado y presente de los vecinos turrialbeños. 
Al finalizar el taller, se concretaron tres intervenciones en esta línea:

EI regreso de las pericas. El primer espacio seleccionado para intervenir fue el Parque Central de Turrialba, el cual, como contaron los estudiantes, estaba poblado de perezosos, los cuales interactuaban de forma constante con los visitantes del lugar. Aunque en la memoria del lugar siguen presentes, hoy en día ya no hay "pericas" - como le dicen coloquialmente los turrialbeños-, debido a que empezaron a correr riesgos y a disminuir su población como consecuencia de los cambios urbanos que empezó a sufrir Turrialba en las últimas décadas.

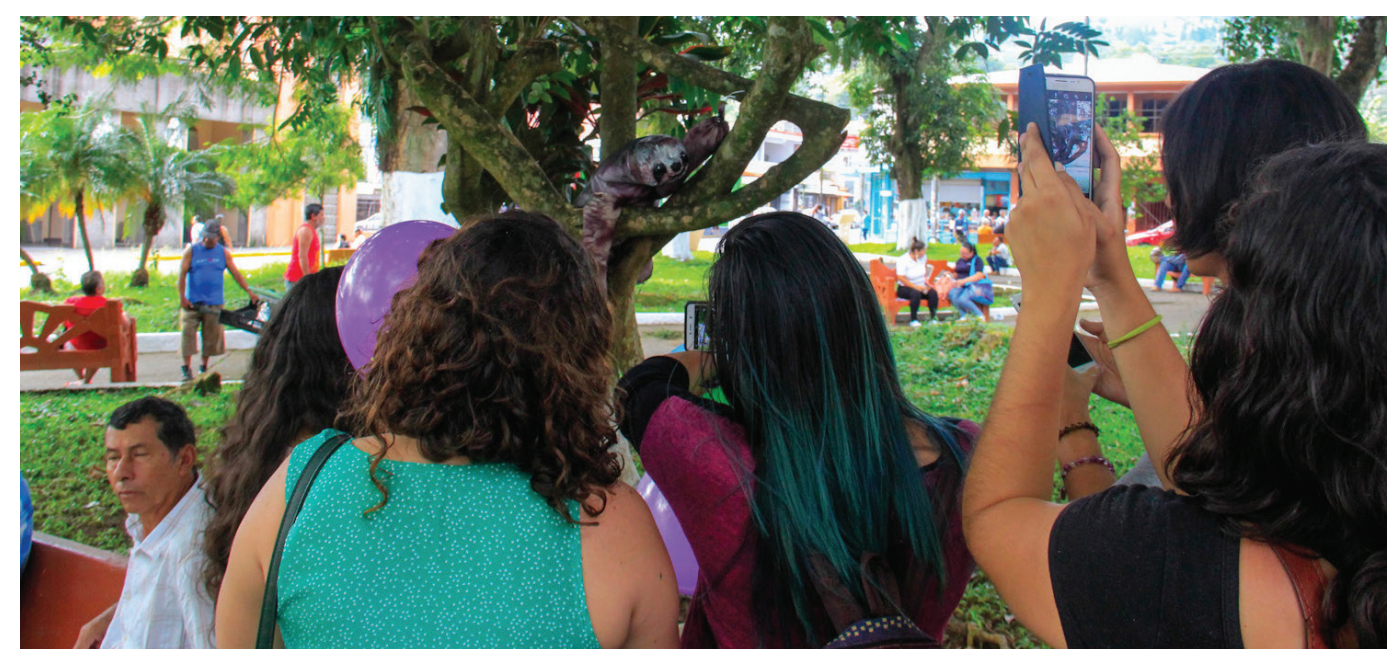

Foto \#7:

Taller Análisis, Experimentación e intervención del espacio publico. (Pericas) (C) Olar Zapata

En respuesta a esta situación, la intervención ejecutada por el grupo de estudiantes consistió en la fabricación de un maniquí a escala real de un perezoso, el cual se situó en uno de los árboles del Parque Central para generar memoria e incentivar la discusión sobre su erradicación en ese entorno urbano. Desde el momento de su colocación, como efecto directo se constató cómo las personas en el Parque Central comenzaron a recuperar esa historia y, sobre todo, a preguntarse sobre la identidad turrialbeña. Pero, además de la intervención en el parque, el grupo de estudiantes también se encargó de correr el rumor del posible regreso de las "pericas" al lugar en las redes sociales, lo cual amplió el rango de la intervención a otro tipo de esfera pública. 
Los globos de la suerte. El segundo grupo de estudiantes, en su análisis de la vida turrialbeña, identificó cómo la cohesión social entre vecinos se estaba perdiendo al consolidarse el centro de Turrialba como una ciudad comercial y, en gran medida, caótica. Más aún, se evidenció cómo esos cambios profundizaban la distancia entre clases, género, edad e incluso raza, características que, aunque coincidían en el espacio urbano, se alejaban, pues sus relaciones se limitaban a los intercambios mercantiles.

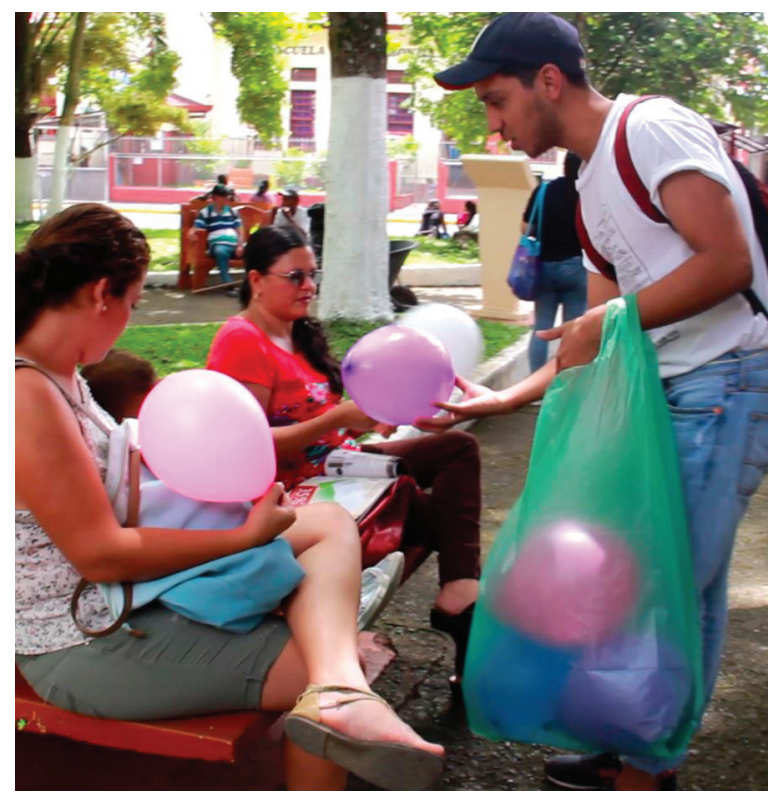

Foto \#8:

Taller Análisis, Experimentación e intervención del espacio publico. (Globos)

colar Zapata

A partir de este análisis, los estudiantes propusieron una intervención que consistió en regalar aproximadamente doscientos globos que contenían en su interior frases positivas a modo de regalo entre personas desconocidas que se abrían y revelaban al estallar cada globo. Este proyecto, dada la visualidad y simbolismo lúdico del globo, pretendía fortalecer la empatía entre desconocidos y disminuir, aunque sea por un momento, la distancia entre las personas de la ciudad. Como comentan Göbel y Zapata (2017) en el informe presentado del taller: "Al ser globos de colores, y al tener una actitud cercana y amable, las personas los recibían los regalos con gusto dejando en cada caso escapar ligeras sonrisas y miradas de desconcierto y complicidad". 
En ese sentido, se podría decir que este proyecto, siguiendo los postulados de Hardty y Negri (2004), permitió crear formas biopolíticas de intercambios generosos basados en la producción de saberes y afectos inmateriales. Entre los mensajes escondidos en los globos estaban, por ejemplo, los siguientes: “¡No te rindas! Continúa intentándolo, lo mejor está por llegar", "Quien controla los medios controla la mente" o "Regala un abrazo y pídele a alguien más que regale otro abrazo".

La brigada repara puentes. Por último, este proyecto consistió en una intervención "médica" en el Puente Blanco de Turrialba, el cual presenta múltiples daños que atentan con la seguridad de los peatones que lo transitan.

Los estudiantes propusieron visualizar todas sus heridas vendándolas como si de una paciente se tratara. Además, se identificaron y documentaron todos sus desperfectos, grietas y roturas (51 en total) señalándolas con pintura roja que simulaba sangre.

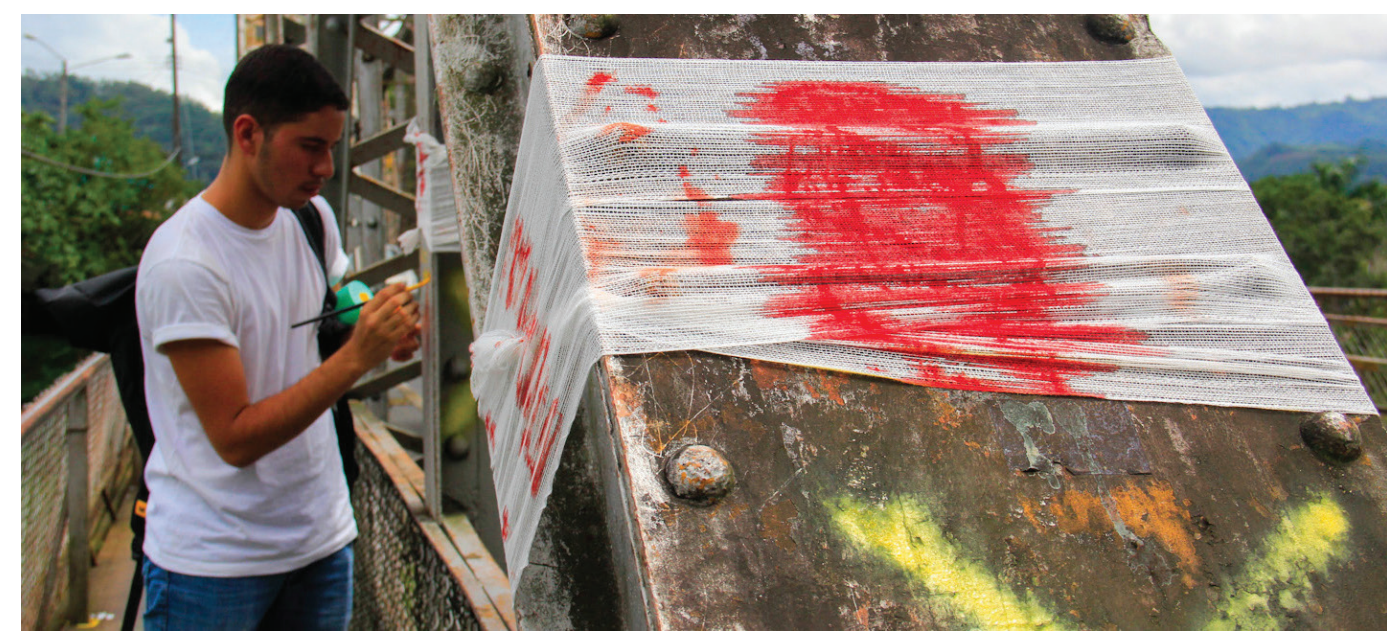

Foto \#9:

Taller Análisis, Experimentación e intervención del espacio publico. (Brigada reparapuentes)

COlar Zapata 
Por último, se colocaron frases dichas por la personas que recorrían el puente. Así pues, quedaron plasmadas palabras como: "Se cae pa bajo", "Puente en mal estado", “¡Cuidado se corta!”, “¡Ojo!”. De esta manera, desde el momento de la intervención los vecinos ya se sumaban a la dinámica, ya que reclamaban el saneamiento real del puente. En redes sociales, la gente también comentó el proyecto y proliferó las denuncias que reforzaban y aplaudían el trabajo de la Brigada reparapuentes.

\section{Conclusiones}

Así, en suma, estas tres intervenciones articuladas — una centrada en la memoria e identidad turrialbeña, otra en las relaciones interpersonales de los vecinos y, la última, de carácter performático, con un claro acento crítico dirigido a los entes de gobierno- evidenciaron el amplio espectro de conflictos y problemáticas que envuelven la condición pública de un centro urbano dinámico en constate cambio. El recinto Universitario de Turrialba, como un núcleo educativo, cultural y deportivo relevante para la comunidad turrialbeña, no puede desconocer esta situación. En cambio, debe comprometerse como un espacio potencial para impulsar y profundizar transformaciones en su entorno cercano, consiente de su capacidad y responsabilidad para intervenir positivamente en él.

Respecto al mural Parcelas de Aire, este artículo ha pretendido sistematizar el proyecto acentuando su carácter transdisciplinar y resaltando no sólo aquellos aspectos normativos e imprescindibles relativos al desarrollo y la descripción de sus propósitos, sino, además, un conjunto de fundamentaciones que se diversifican en diferentes áreas disciplinares: arqueológicas, históricas, filosóficas, artísticas, políticas e, incluso, de gestión institucional. Esta cuestión acentúa su condición como un dispositivo simbólico que va más allá de la ornamentación o la transformación del paisaje. 
Además, ha pretendido presentar el proyecto como antecedente fundamental que, interrelacionado con la consolidación de la carrera de Diseño Gráfico, hizo posible poner sobre el tapete la discusión compleja sobre los entornos de aprendizaje universitarios, entendidos como una comunidad que debe abrirse en espiral hacia su entorno. He ahí la articulación que en este artículo hemos considerado indispensable y que muestra significativamente el trabajo de casi tres años de coordinación entre diversas unidades académicas y sedes, donde se ha tratado al máximo de romper con el carácter centralista de la universidad, mediante el fortalecimiento de la misma sede, ahora en dirección contraria, desde su relación con el entorno inmediato.

\section{Notas}

1. La traducción es mía.

2. La traducción es mía.

\section{Referencias}

Aguilar, C. (1972). Guayabo de Turrialba. Arqueología de un sitio indígena prehispánico. San José: Editorial Costa Rica.

Arias, A. C., Castrillo, F. y Herrera, G. (2012). Una historia escrita en piedra: Petrograbados en Guayabo Turrialba. San José: UCR-VAS.

Debravo, J. (2012). Obra poética. San José: Editorial de Costa Rica.

Fals Borda, O. (2003). Ciencia, compromiso y cambio social. Buenos Aires: El Colectivo.

Göbel, C. y Zapata, O. (2017). Informe administrativo del taller Análisis, experimentación e intervención del espacio público. México D.F.: UAM-A. 
Arte público en la Sede del Atlántico...

Freire, P. (1998). ¿Extensión o comunicación?: La concientización en el medio rural. Buenos Aires: Siglo XXI Editores.

Hardty, M. y Negri, A. (2004). Multitud: Guerra y democracia en la era del imperio. Barcelona: Debate.

Krauss, R. (1996). El campo expandido de la Escultura. En La originalidad de las vanguardias y otros mitos modernos. Madrid: Akal.

Lefebvre, H. (1978). El derecho a la ciudad. Barcelona: Península.

Lacy, S. (1996). Mapping the terrain. New genre public art. Washington: Bay Press. 